\title{
Implante de Stent dentro de Stent Recém Implantado em Ponte de Veia Safena para Otimização do Resultado Angiográfico
}

\author{
A ntonio Esteves Fo, Celso Kiyochi Takimura, Evandro Costa Lira, Luiz Junya Kajita, Siguemituzo Arie, \\ Giovanni Bellotti, Fulvio Pileggi
}

São Paulo, SP

Mulher de 60 anos, com angina progressiva e revascularização do miocárdio, há oito anos, com ponte de veia safena para coronária direita e anastomose de artéria mamaria esquerda para artéria descendente anterior. Submetida a implante de stent Gianturco-Roubin II em terço proximal da ponte de veia safena para artéria coronária direita, com resultado insatisfatório pela persistência de lesão residual, provavelmente, decorrente de prolapso para dentro da luz de material aterosclerótico através dos coils. Foi implantado outro stent (PalmazSchatz biliar) dentro do stent GRII com sucesso e ótimo resultado angiográfico. Um $2^{\circ}$ stent Palmaz-Schatz biliar foi implantado em lesão distal no corpo da ponte, ultrapassando os dois stents, anteriormente implantados, com sucesso. Em algumas situações, implante de stent dentro de outro stent é recurso útil para otimização de resultado angiográfico do implante de um stent.

\section{Stenting a Stent in Saphenous Vein Graft to Optimize theAngiographic Result}

A 60 year-old woman with progressive angina who had been submitted to saphenous bypass-graft to right coronary artery and a left mammary artery graft to anterior descending artery eight years previously, underwent implantation of a Gianturco Roubin II stent in the proximal third of the saphenous vein graft. The result was suboptimal by persistence of a residual stenosis probably due to prolapse of atherosclerotic material through the coil spaces. Another stent (Palmaz-Schatz biliar stent) was implanted at the previously stented site with no residual stenosis. Another Palmaz-Schatz biliar stent was successfully implanted in the distal body of the graft to treat another lesion (passing through the previously stents without difficulty). Stenting a stent, in selected situations, is a useful tool to optimize the angiographic result of stent implantation.

Arq Bras Cardiol, volume 70 (nº 3), 177-179, 1998

Após a documentação da superioridade do emprego do stent intracoronário Palmaz-Schatz (PS) (Johnson \& Johnson Interventional Systems, Warren, $\mathrm{NJ}$ ) em relação à dilatação com balão, para tratamento de lesões coronárias selecionadas, o implante dessas endopróteses em todo o mundo expandiu-se de forma extraordinária. Atualmente, são utilizados, após resultado insatisfatório com angioplastia coronária com balão (retração elástica importante, dissecção com iminência de oclusão e oclusão aguda) e na tentativa de se obter um ótimo resultado imediato e evolução a longo prazo sem reestenose coronária (prevenção de

Instituto do Coração do Hospital das Clínicas - FMUSP

Correspondência: Antonio Esteves $F^{\circ}$ - Rua Oscar Freire, 264 -10 - 01426-001 São Paulo, SP

Recebido para publicação em 22/4/97

Aceito em 7/1/98 reestenose), em artérias nativas e enxertos arteriais e venosos ${ }^{1}$.

Vários são os tipos de endopróteses disponíveis e com diferentes estruturas, materiais constituintes, configurações, propriedades físicas e o mais importante, limitações inerentes a cada modelo.

Relatamos uma forma de otimização de um resultado angiográfico insatisfatório, com o implante de um stent dentro de um outro recém implantado.

\section{Relato do Caso}

Mulher de 60 anos, com sintoma de angina progressiva há um mês da internação. Antecedente de revascularização do miocárdio em 15/2/89 com realização de ponte de veia safena para a artéria coronária direita (PVS-CD) e anastomose da artéria mamária esquerda em artéria descendente anterior (MaE-DA). Submetida à cinecoronariografia 
que revelou artéria coronária direita e descendente anterior ocluídas e artéria circunflexa com irregularidades parietais. A PVS-CD apresentava obstrução proximal e excêntrica de $90 \%$ e obstrução de $50 \%$ no terço distal (fig. 1A). A MaEDA não apresentava lesões obstrutivas.

Decidimos por intervenção na PVS-CD (diâmetro de referência de $3,1 \mathrm{~mm}$ ) com implante de stent. Através de acesso arterial braquial direito (insuficiência arterial em membros inferiores), cateterizamos, seletivamente, a PVSCD com cateter guia Judkins Right (JR4) (Cordis Corporation) $8 \mathrm{~F}$. Posicionamos a corda guia 0,014 polegadas, Traverse ${ }^{\mathrm{TM}}$ (ACS, Santa Clara, CA) no leito distal da coronária direita e realizamos dilatação com duas insuflações com cateter balão 3,0x20mm Lifestream ${ }^{\mathrm{TM}}$ (ACS, Santa Clara, CA) com pressão de insuflação de 6 ATM (fig. 1B). A seguir, implantamos um stent GianturcoRoubin II (GRII) (Cook Inc. Co.) 3,5x20mm na obstrução proximal, liberando-o com 6 ATM (fig. 1C). Realizamos, em seguida, dilatação intra-stent, com o mesmo balão empregado na pré-dilatação, com pressão final de 18 ATM, permanecendo uma lesão residual intra-stent, de aspecto irregular (fig. 1D), avaliada pela angiografia coronária quantitativa (ACQ) on line (DCI - Philips) em 28\%. Não havendo melhora angiográfica após várias insuflações adicionais (fig. 2A), realizamos implante de outro stent, PS “biliar” (P204), montado no mesmo balão, dentro do stent GRII (stent sobre stent), com pressão máxima de insuflação de 20 ATM (fig. 2B). A lesão distal na PVS-CD, inicialmente, estimada com obstrução de $50 \%$, apresentava estenose de maior importância $(70 \%)$, sendo tratada com sucesso com implante de

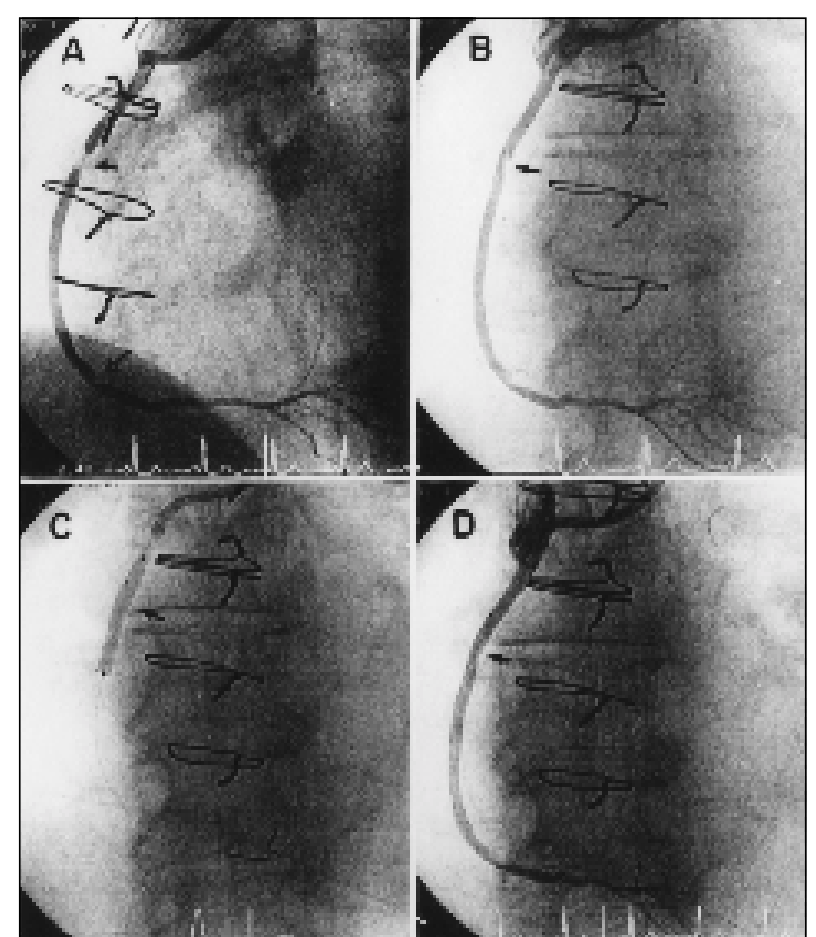

Fig. 1 - A) PVS-CD com obstrução crítica no terço proximal e de grau moderado no terço distal (setas); B) resultado angiográfico após dilatação com balão da obstrução do terço proximal (seta); C) implante de stent GRII (seta); D) resultado após implante de stent GRII mostrando lesão residual intra-stent (seta). outro stent PS “biliar” (P204), ultrapassando sem dificuldade os dois stents, previamente, implantados (fig. 2C). Injeção de contraste final mostrou excelente resultado do procedimento (fig. 2D), confirmado pela ACQ.

A paciente foi medicada com ticlopidina $(500 \mathrm{mg} / \mathrm{dia}) \mathrm{e}$ ácido acetil-salicílico (100mg/dia) e recebeu alta hospitalar no dia seguinte ao procedimento. Com três meses de seguimento, a paciente encontra-se assintomática, e com reestudo angiográfico planejado para o $6^{\circ}$ mês.

\section{Discussão}

Vários problemas estão relacionados à intervenção por cateter em pontes de veia safena, como embolização distal, no-reflow, reestenose, reoclusão e infarto do miocárdio. Atualmente, lesões complexas nesses enxertos venosos são preferencialmente tratadas com implante de stent. Vários estudos observacionais têm relatado baixos índices de complicações isquêmicas e taxas de reestenose entre 20 a $40 \%$ para lesões de novo ${ }^{2}$.

O stent GRII é um stent, recentemente, desenvolvido (1995), tendo como vantagens em relação ao stent GR da primeira geração ser montado em balão não complacente, ter menor perfil, maior cobertura da parede do vaso, ser indicado para vasos até $5 \mathrm{~mm}$ e possuir marcas radiopacas em suas extremidades. Sua configuração tipo coil torna-o menos indicado para o tratamento de lesões tipo "espícula", "membrana" ou lesões ostiais. Além disso, à medida que este stent é expandido, alargam-se os espaços entre as alças, podendo permitir herniação de material friável para o lúmen, especialmente em vasos calibrosos $(>4 \mathrm{~mm})^{2}$. Provavelmente foi o que ocorreu no presente caso, após implante do stent GRII em ponte de veia safena.

A experiência com a utilização de stent GR em ponte de veia safena é limitada. A maior experiência relatada é de Bilodeau e col $^{3}$ que trataram 37 pacientes, não observando óbitos relacionados ao procedimento e angiografia de controle revelando índice de reestenose de $35 \%$.

Implante de stent dentro de outro stent é pouco relatado na literatura, sendo bastante restrita suas indicações. Morís e $\mathrm{col}^{4}$, após implante de um stent PS articulado de $15 \mathrm{~mm}$ em artéria circunflexa, observaram, após quatro meses, reestenose focal em ponto correspondente à articulação do stent. Trataram essa reestenose com dilatação com balão, observando, entretanto, dissecção importante intra-stent, com lentificação do fluxo coronário e dor precordial. Apesar de redilatação prolongada (10min) não houve melhora, sendo implantado outro stent PS dentro do anterior com correção da dissecção e ótimo resultado final. Realizado reestudo angiográfico com três meses, foi observada manutenção do resultado final da intervenção.

Ghannem $\mathrm{Me} \mathrm{col}^{5}$, quase simultaneamente a Moris e $\mathrm{col}^{4}$ e com indicação semelhante, relataram o tratamento de reestenose de dois stents $\mathrm{PS}$ com implante de mais dois stents PS dentro dos anteriores, com boa evolução clínica hospitalar e a longo prazo (seis meses).

Ceceña ${ }^{6}$ tratou uma reestenose de stent GR implanta- 


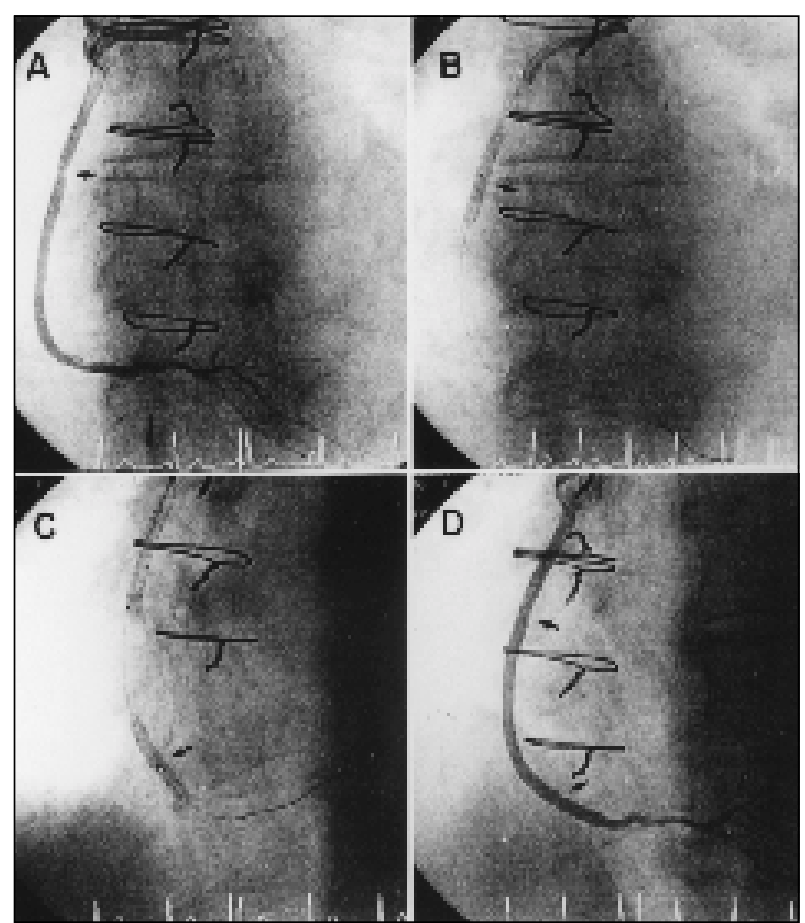

Fig. 2 - A) Resultado angiográfico após várias insuflações com altas pressões mostrando estenose residual intra-stent (seta); B) implante do stent PS "biliar" dentro do stent GRII (seta); C) implante de outro stent PS “biliar" em lesão distal na PVS-CD (seta) ultrapassando os dois stents proximais; D) injeção de contraste final mostrando PVS-CD com excelente resultado angiográfico para os dois locais tratados (setas).

do em ponte de veia safena para a artéria descendente anterior com o implante eletivo (intra-stent) de três stents PS "biliares" (P104). O reestudo com seis meses mostrou perviabilidade do vaso, sem reestenose.
Portanto, nos casos anteriormente publicados, a indicação do implante de stent dentro de outro stent foi para tratamento de reestenose intra stent. No nosso caso, a indicação do implante do stent PS foi para otimizar o resultado angiográfico imediato, após o implante de um stent (GRII). Esta estratégia permitiu um ótimo resultado final, provavelmente, por contenção de placa aterosclerótica entre a malha do stent tubular (PS) e a parede do vaso. Neste caso, apesar de não termos empregado ultra-som, a expansão completa dos stents foi confirmada por ACQe, indiretamente, evidenciada pela fácil ultrapassagem de outro stent PS para implante em lesão distal no corpo da PVS-CD. A evolução a longo prazo, baseada nos casos previamente relatados ${ }^{4-6}$, provavelmente será satisfatória.

Outra indicação para aplicação desta técnica é a recomendada por Dean Kereiakes ${ }^{7}$ quando, após implante de stent em lesão localizada em óstio, observa-se recoil e resultado insatisfatório. Considera esta estratégia como sendo a única forma efetiva de aumentar a força radial do stent, contrapondo-se, assim, ao recolhimento elástico.

Concluímos que, com o aumento das indicações de implante de stent, utilizando-se diferentes modelos no tratamento de obstruções coronária, cada vez mais complexas, surgem novas situações que exigem soluções criativas e eficientes. Este relato de caso acrescenta mais uma indicação para implante de stent dentro de outro stent.

\section{Agradecimentos}

À Sra Mitsuko Oshiro Mori e ao Sr Sebastião José dos Santos pelo auxílio no preparo do material fotográfico.

\section{Referências}

1. Pepine CJ, Holmes DR - Coronary Artery Stents - ACC Expert Consensus Document. J Am Coll Cardiol 1996; 28: 782-94.

2. Wong SC, Leon MB - Stent placement for saphenous vein graft disease. In: Sigwart U, ed - Endoluminal Stenting. London: WB Saunders, 1996: 428.

3. Bilodeau L, Iyer S, Cannon ADetal-Flexible coil stent(Cook Inc.) in saphenous vein grafts: clinical and angiographic follow-up. J Am Coll Cardiol 1992; 19: 264A.

4. Morís C, Alfonso F, Lambert Jl et al - Stenting for coronary dilation of in-stent restenosis: Stenting a previously stented site. Am Heart J 1996; 131: 834-6.
5. Ghannem M, Lefèvre T, Bernard A, Philippe $\mathrm{J}$ - Resténose sur endoprothèse coronaire: traitement par implantation d'une nouvelle endoprothèse: à propos d'un cas. Ann Cardiol Angeiol 1996; 45: 287-90.

6. Ceceña FA - Stenting the stent: Alternative strategy for treating in-stent restenosis. Cathet Cardiovasc Diagn 1996; 39: 377-82.

7. Kereiakes D- Ostial lesion: calcified RCA. In: Safian RD, Freed M. eds - Tough Calls in Interventional Cardiology. An Instructional Atlas. Birmingham, Michigan: Physicians' Press, 1997: 60. 